\title{
Impacto de la resistencia a antimicrobianos y de serotipos de Streptococcus pneumoniae en la mortalidad de niños menores de 5 años con enfermedad invasora ${ }^{1}$
}

\author{
Ana María Ríos, ${ }^{2}$ Fernando de la Hoz, ${ }^{2}$ Aura Lucía Leal, ${ }^{2}$ \\ Orlando Castillo, ${ }^{2}$ Elizabeth Castañeda ${ }^{2}$ y Grupo Colombiano \\ de Trabajo en Streptococcus pneumoniae ${ }^{3}$
}

RESUMEN Las enfermedades invasoras ocasionadas por Streptococcus pneumoniae han sido por mucho tiempo una causa importante de mortalidad. Si bien se sabe que la resistencia de S. pneumoniae a la penicilina y otros antimicrobianos se ha incrementado con el tiempo, aún no se ha esclarecido el efecto de este cambio sobre la mortalidad. Se desconoce igualmente el impacto de la virulencia de los tipos capsulares del microorganismo en la mortalidad. El objetivo de este estudio de cohorte retrospectivo fue determinar los factores de riesgo de mortalidad por enfermedad neumocócica en niños menores de 5 años. Durante un estudio de serotipificación de S. pneumoniae patrocinado por el Sistema Regional de Vacunas de la OPS se revisaron con este fin las fichas epidemiológicas de 245 pacientes de esa edad en quienes se diagnosticó enfermedad invasora por S. pneumoniae entre 1994 y 1996 en Colombia. De los 245 pacientes, 29 (11\%) fallecieron. En el análisis univariado no se establecieron diferencias significativas entre los pacientes que murieron $y$ aquellos que sobrevivieron en cuanto a edad, sexo, procesos patológicos subyacentes al ingresar, o concordancia del tratamiento antimicrobiano recibido. Las variables que se asociaron con la mortalidad fueron un diagnóstico de meningitis; infección por S. pneumoniae resistente a la penicilina, trimetoprima-sulfametoxazol (TMS) o eritromicina; multirresistencia, y los tipos capsulares $6,23 F, 7 F, 8$ y 35 B. En el análisis por regresión logística siguieron mostrando asociación con la mortalidad los tipos capsulares $7 F$ (razón de posibilidades $u$ odds ratio $[O R])=7,13$; $\mathrm{P}=0,04)$ y $8(\mathrm{OR}=13,8 ; \mathrm{P}=0,07)$, la polipnea $(\mathrm{OR}=2,74 ; \mathrm{P}=0,03)$, el diagnóstico de meningitis $(\mathrm{OR}=5,02 ; \mathrm{P}=0,0001)$ y la resistencia a TMS $(\mathrm{OR}=2,62 ; \mathrm{P}=0,02)$. En los casos de neumonía, el factor que más se asoció con la mortalidad fue el tipo capsular, mientras que en los casos de meningitis, dicho factor fue la resistencia a antimicrobianos. En el desarrollo de una vacuna deberian tenerse en cuenta las diferencias de mortalidad según los serotipos, a fin de lograr un mayor impacto en la morbilidad y mortalidad infantiles por enfermedad de origen neumocócico.

Streptococcus pneumoniae es la principal causa bacteriana de enfermedad

\footnotetext{
1 La información analizada en este trabajo forma parte del Proyecto Latinoamericano de Serotipificación de Streptococcus pneumoniae, coordinado desde la sede de la Organización Panamericana de la Salud en Washington, D.C., Estados Unidos de América, y cofinanciado por el Organismo Canadiense para el Desarrollo Internacional (CIDA).
}

broncopulmonar adquirida en la comunidad, así como también de otitis media aguda, sinusitis y meningitis $(1,2)$. No

\footnotetext{
2 Instituto Nacional de Salud, Grupo de Microbiología, Santa Fe de Bogotá, Colombia. Dirección postal: Avenida El Dorado, carrera 50, Santa Fe de Bogotá, Colombia.

3 La lista de participantes aparece en el anexo 1.
}

obstante los adelantos en el tratamiento con antimicrobianos y en el desarrollo de vacunas, tanto para la neumonía neumocócica grave como para la meningitis, estas enfermedades siguen asociándose con altas tasas de mortalidad (3). Se ha estimado que más de un millón de niños mueren todos los años por neumonía neumocócica y aproxi- 
madamente la mitad son menores de 1 año (4).

A partir de 1967, año en que se describió en Australia el primer aislamiento de $S$. pneumoniae con susceptibilidad disminuida a la penicilina, la resistencia de esas bacterias a los medicamentos se ha incrementado y diseminado por todas partes del mundo. La prevalencia de cepas resistentes es alta, especialmente en España, Hungría y Sudáfrica $(5,6)$. Hasta el momento no se ha logrado establecer una clara asociación entre la resistencia a antimicrobianos y la mortalidad y, en cambio, se han considerado más importantes el estado crítico del paciente al momento del diagnóstico y la presencia de procesos patológicos subyacentes (7-10).

De los 90 tipos capsulares de S. pneumoniae descritos, solo 12 se han asociado frecuentemente con enfermedad invasora (11). Existen pocos informes de estudios en los que se haya intentado determinar la posible asociación entre los serotipos y la mortalidad (12-14), pese a que serían especialmente útiles con el fin de desarrollar una vacuna conjugada que sea inmunógena en niños menores de 5 años. Hasta ahora, el criterio de selección de los serotipos incluidos en las vacunas ha sido la frecuencia con que han sido aislados (15). Sin embargo, se ha observado que los serotipos presentan diferencias tanto en inmunogenicidad como en el tipo de enfermedad que producen (14, 16). Por tal razón cabe pensar que no es acertado emplear la frecuencia de los tipos capsulares como único criterio para producir una vacuna con impacto adecuado, tanto en la gravedad de la enfermedad por S. pneumoniae como en la mortalidad que produce.

De 1994 a 1996 se realizó en Colombia, como parte del proyecto del Sistema Regional de Vacunas (SIREVA) de la OPS, un estudio de serotipificación de $S$. pneumoniae causante de enfermedad invasora en una cohorte de niños menores de 5 años (17). Ese estudio se llevó a cabo en Santa Fe de Bogotá, Medellín y Cali con la participación de centros asistenciales. El objetivo de este trabajo fue analizar los datos obtenidos en ese estudio para determinar los factores asociados con la morta- lidad, desde el punto de vista de la influencia ejercida por los tipos capsulares de $S$. pneumoniae y por la resistencia de esta bacteria a los antimicrobianos.

\section{MATERIALES Y MÉTODOS}

El presente trabajo se diseñó como un estudio de cohorte retrospectivo basado en la revisión de las fichas de los pacientes del estudio mencionado. Se escogieron como muestra los expedientes de $245(75,6 \%)$ pacientes, que contenían información sobre su evolución clínica, y se excluyó a los casos de sepsis debido a la falta de homogeneidad de su definición en los centros colombianos que participaron en el estudio. Un análisis preliminar permitió comprobar que en los 245 pacientes de esta cohorte no había diferencias estadísticamente significativas en la distribución por edad, sexo y diagnóstico de los pacientes, ni en los tipos capsulares y resistencia a antimicrobianos de sus aislados, con respecto al total de los 324 pacientes originales.

El estudio se llevó a cabo principalmente en centros asistenciales de las ciudades de Santa Fe de Bogotá, Medellín y Cali (anexo 1), aunque una pequeña proporción de los pacientes provino de otras ciudades, como Cúcuta y Bucaramanga. La mayoría de los centros de atención fueron hospitales universitarios que atendían a niños de bajo nivel socioeconómico.

De las fichas epidemiológicas se obtuvieron datos sobre edad, sexo, peso, examen físico al ingreso, diagnóstico, procesos patológicos subyacentes, toma de antibióticos previos, tratamiento antimicrobiano prescrito y evolución clínica de cada paciente. Además, se incluyeron los datos del laboratorio de microbiología del Instituto Nacional de Salud referentes a los tipos capsulares y a la susceptibilidad a antimicrobianos de las bacterias aisladas, esta última establecida de acuerdo con los criterios del National Committee for Clinical Laboratory Standards [Comité Nacional de Normas Clínicas para Laboratorios de los Estados Unidos de América] (10). Se consideraron multirresistentes los ais- lados resistentes a tres o más familias de agentes antimicrobianos (6).

En lo que se refiere a definiciones, se emplearon las de los cuadros clínicos del estudio previo (17). La enfermedad invasora se determinó sobre la base de los diagnósticos clínicos de meningitis, sepsis, artritis y peritonitis, y el diagnóstico de neumonía se basó en los criterios de la OMS (17). En cuanto a los síntomas de los pacientes al ingreso, la polipnea se definió de acuerdo con los límites propuestos para la edad (18) y la hipotermia como una temperatura corporal de $36,5^{\circ} \mathrm{C}$ o menos. En los pacientes de la muestra se detectaron cardiopatía congénita, enfermedad pulmonar crónica, síndrome nefrótico, cáncer, síndrome de Down y trauma craneocerebral como estados patológicos subyacentes (19).

Todos los pacientes se clasificaron en tres categorías, de acuerdo con la susceptibilidad de los aislamientos individuales de $S$. pneumoniae al tratamiento antimicrobiano prescrito, a saber: 1) concordante, si la bacteria aislada del paciente era sensible al menos a uno de los antibióticos de elección para el tratamiento de la infección neumocócica y ese antibiótico se incluyó en el esquema prescrito; 2) discordante, cuando el aislamiento era resistente a todos los antibióticos de elección que se incluyeron en el esquema antimicrobiano prescrito; y 3) desconocido, cuando no se pudo obtener esa información. Además, dadas las observaciones de estudios previos (6) que muestran el impacto de la resistencia intermedia en la evolución desfavorable de la meningitis, en la clasificación concordante se incluyó el criterio de que los aislamientos presentaran resistencia tanto intermedia como alta.

La asociación de los diferentes factores (diagnóstico clínico, procesos patológicos subyacentes, síntomas detectados por el examen clínico en el momento del ingreso, resistencia a antimicrobianos y tipos capsulares de los aislamientos) con la mortalidad se evaluó calculando los riesgos relativos y los intervalos de confianza de 95\% (20) con el programa Epi Info, versión 6.03 (21). Para analizar la asociación de los diferentes serotipos con la mortalidad, 
se tomó como grupo de referencia al de los tipos capsulares relacionados con una mortalidad menor de $10 \%$. La asociación entre la resistencia a antimicrobianos y la mortalidad se estratificó por diagnóstico. Asimismo, se estratificó por resistencia a antimicrobianos y diagnóstico la asociación de los serotipos con la mortalidad.

Al determinar la significación estadística, la prueba de ji al cuadrado $\left(\chi^{2}\right)$ se usó para comparar proporciones y el estadístico $t$ de Student para comparar medias.

En la regresión logística se tomó como variable dependiente la mortalidad. Las variables independientes fueron la edad, el sexo, un diagnóstico de meningitis, algunos hallazgos del examen físico de ingreso como las frecuencias cardíaca y respiratoria, la temperatura, el peso, el tiraje y la cianosis, así como la presencia de procesos patológicos subyacentes; los serotipos asociados con una mortalidad mayor de $10 \%$, la resistencia a antimicrobianos y la multirresistencia; y la concordancia del tratamiento recibido. La inclusión de estas variables en el modelo dependió de su significación estadística en el análisis univariado y la magnitud de su asociación con la variable dependiente. Los criterios empleados fueron que, en el análisis bivariado, presentara un riesgo relativo (RR) $<0,7$ o $>1,5$, o una asociación con un valor $P<0,25$, o un efecto dosis-respuesta. También se tuvo en cuenta la relevancia biológica de variables que, aunque no estuvieran asociadas estadísticamente, pudieran incluirse por plausibilidad biológica, así como el control de posibles fuentes de confusión. Complementariamente se empleó el método stepwise de eliminación retrospectiva para comparar los modelos obtenidos. La regresión logística se realizó con el programa SPSS, versión 6.1 (22).

\section{RESULTADOS}

\section{Datos demográficos, de procedencia y clínicos}

Los 245 pacientes estudiados se distribuyeron por sexo en $82(33,5 \%) \mathrm{mu}-$ jeres y $163(66,5 \%)$ varones y, por edad, entre 0 y 68 meses, con una media de 16 y una mediana de 12 meses. Según el lugar de procedencia, 135 (55,1\%) pacientes eran de Santa Fe de Bogotá, $73(29,8 \%)$ de Medellín, 34 (13,9\%) de Cali y $3(1,2 \%)$ de otras ciudades. El diagnóstico clínico señaló 124 casos de neumonía, 104 de meningitis y 14 otros diagnósticos: 8 casos de peritonitis y 6 de artritis; además, en dos casos se realizaron biopsias de tejidos blandos y en uno, biopsia de fémur.

De los 245 pacientes, 29 (11,8\%) murieron como resultado de complicaciones de la enfermedad invasora, incluidas $13 / 82(15,9 \%)$ de las niñas y $16 / 163$ $(9,8 \%)$ de los varones $(P=0,16)$. Por grupos de edad, fallecieron 18/127 $(14,2 \%)$ menores de 1 año y 11/118 $(9,3 \%)$ mayores de esa edad $(P=0,24)$.

La letalidad por diagnóstico se distribuyó de la siguiente manera: 20/104 $(19,2 \%)$ fallecieron de meningitis, $8 / 124(6,4 \%)$ de neumonías y 1 de peritonitis $(5,9 \%)$ de los 14 cuadros clasificados como otros diagnósticos $(P=$ $0,008)$. El riesgo de morir fue tres veces más alto en los pacientes con meningitis que en los que tuvieron otros diagnósticos.

En el análisis bivariado, ninguno de los hallazgos del examen físico de ingreso (polipnea, hipotermia y peso inferior al percentil 50 para la edad) se asoció de forma significativa con la mortalidad. En $157(64,1 \%)$ de los 245 pacientes estudiados se obtuvo información sobre la presencia o ausencia de procesos patológicos subyacentes. De los $80(50,9 \%)$ que los presentaron, $11(13,7 \%)$ fallecieron, mientras que de los $77(49,0 \%)$ que no los presentaron, fallecieron 6 (7,8\%); RR = 1,79; IC95\%: $0,70$ a 4,59$)$.

\section{Mortalidad y resistencia a antimicrobianos de los aislamientos de Streptococcus pneumoniae}

En el cuadro 1 se observa la distribución de la mortalidad asociada con la resistencia y multirresistencia a los antimicrobianos. Los pacientes de quienes se obtuvieron aislamientos resistentes a la penicilina tuvieron una probabilidad más alta de morir $(22,6 \%)$ que aquellos con aislamientos sensibles a ese medicamento $(11,5 \%)(\mathrm{RR}=$ 2,20; IC95\%: 1,02 a 4,71). La asociación de la resistencia a la penicilina con la mortalidad se mantuvo al comparar los aislamientos muy resistentes con los sensibles, excluyendo a los intermedios, y al comparar los aislamientos con resistencia intermedia con los sensibles ( $R R=2,21$; IC95\% 0,93 a 5,26). Sin embargo, la asociación disminuyó al comparar los aislamientos muy resistentes con los de resistencia intermedia y los sensibles, como un solo grupo $(R R=1,94$; IC95\%: 0,54 a 6,94).

Se encontraron asociaciones con la mortalidad en los pacientes con aislamientos resistentes a TMS y eritromicina, pero la mortalidad no se asoció con resistencia a la ceftriaxona ni al cloranfenicol (cuadro 1). En los aislamientos de los pacientes que fallecieron, todos los que presentaron susceptibilidad disminuida a la penicilina y eran resistentes a la ceftriaxona y a la eritromicina presentaron multirresistencia.

En la mortalidad por meningitis se observó un efecto dosis-respuesta correspondiente a los diferentes grados de susceptibilidad a la penicilina. Así, en los pacientes con aislamientos sensibles, la mortalidad fue de $17 \%$, pero esta ascendió a $23 \%$ en los que tuvieron aislamientos con resistencia intermedia y a $66,6 \%$ en pacientes con aislamientos muy resistentes $(P=0,06)$. En los casos de neumonía no se observó ese patrón $(P=0,59)$ (cuadro 2).

El riesgo relativo de morir de meningitis fue similar al comparar los aislamientos muy resistentes con los intermedios y los sensibles como un solo grupo. Ese riesgo disminuyó y perdió significación estadística cuando los pacientes con aislamientos muy resistentes e intermedios se consideraron como un solo grupo $(31,2 \%$ frente a 17\%; RR = 1,83; IC95\%: 0,78 a 4,34).

En los pacientes con neumonía y aislamientos muy resistentes a la penicilina no hubo fallecimientos. Sin embargo, se observó un riesgo mayor de morir en los pacientes que presentaron susceptibilidad disminuida a la penicilina $(14,2 \%)$ que en los que tuvieron aislamientos sensibles a este agente 
CUADRO 1. Asociación de la resistencia a antimicrobianos con la mortalidad en niños menores de 5 años con enfermedad invasora por Streptococcus pneumoniae, Colombia, 1994-1996

\begin{tabular}{|c|c|c|c|c|c|}
\hline \multirow[b]{2}{*}{ Antibiótico } & \multicolumn{2}{|c|}{ Fallecidos } & \multicolumn{2}{|c|}{ Supervivientes } & \multirow[b]{2}{*}{$\mathrm{RR}^{\mathrm{a}}(\mathrm{IC} 95 \%)$} \\
\hline & No. & (\%) & No. & $(\%)$ & \\
\hline \multicolumn{6}{|l|}{ Penicilina } \\
\hline $\mathrm{R}^{\mathrm{b}}$ & 7 & $(22,6)$ & 24 & $(77,4)$ & $2,20(1,02$ a 4,71$)$ \\
\hline $\mathrm{S}^{\mathrm{c}}$ & 22 & $(11,5)$ & 192 & $(88,5)$ & \\
\hline \multicolumn{6}{|l|}{$\mathrm{TMS}^{\mathrm{d}}$} \\
\hline $\mathrm{R}$ & 14 & $(20,5)$ & 54 & $(79,5)$ & $2,43(1,24$ a 4,76$)$ \\
\hline$S$ & 15 & $(8,5)$ & 162 & $(91,5)$ & \\
\hline \multicolumn{6}{|l|}{ Eritromicina } \\
\hline $\mathrm{R}$ & 7 & $(25,0)$ & 21 & (75) & $2,47(1,16-5,24)$ \\
\hline $\mathrm{S}$ & 22 & $(10,1)$ & 195 & $(89,9)$ & \\
\hline \multicolumn{6}{|l|}{ Ceftriaxona } \\
\hline $\mathrm{R}$ & 2 & $(28,6)$ & 5 & $(71,4)$ & $2,52(0,74-8,56)$ \\
\hline $\mathrm{S}$ & 27 & $(11,3)$ & 211 & $(88,7)$ & \\
\hline \multicolumn{6}{|l|}{ Cloranfenicol } \\
\hline $\mathrm{R}$ & 4 & $(11,1)$ & 32 & $(88,9)$ & $0,93(0,34-2,51)$ \\
\hline$S$ & 25 & $(12,0)$ & 184 & $(88,0)$ & \\
\hline \multicolumn{6}{|l|}{ Multirresistencia } \\
\hline$\geq 3$ antibióticos & 5 & $(22,7)$ & 17 & $(77,3)$ & \\
\hline $1-2$ & 11 & $(15,5)$ & 60 & $(84,5)$ & $2,66(1,05-6,73)$ \\
\hline 0 & 13 & $(8,5)$ & 139 & $(91,5)$ & \\
\hline
\end{tabular}

$(5 \%)$, si bien este hallazgo no fue estadísticamente significativo ( $R R=2,62$; IC95\%: 0,58 a 11,74).

En los pacientes con meningitis se observó el efecto de la resistencia a la ceftriaxona en la mortalidad $(100 \%$ frente a $17,6 \%$ ), pero no así en los de neumonía. El efecto de la resistencia a la eritromicina fue también más importante en los casos de meningitis ( $46 \%$ frente a $15,3 \%$ ) que en los de neumonía (6,6\% frente a $6,4 \%$ ) (cuadro 2 ).

Aunque no se encontraron diferencias en la distribución de la resistencia a TMS por diagnóstico, el efecto de esta resistencia en la mortalidad fue más acentuado en los pacientes con neumonías $(12,9 \%$ frente a $4,3 \%)$ que en los pacientes con meningitis $(28,5 \%$ frente a $14,4 \%)$. La resistencia al cloranfenicol no mostró asociación con la mortalidad al analizar por separado cada uno de los diagnósticos.

En los pacientes con meningitis, el riesgo de morir fue tres veces mayor en los que tuvieron aislamientos multirresistentes $(45,4 \%$ frente a $16,1 \%)$ (cuadro 2); pero en los casos de neumonía no hubo fallecimientos en los pacientes con aislamientos multirresistentes. En los pacientes con diagnósticos diferentes de neumonía y meningitis no se detectó ninguna asociación de la resistencia a antimicrobianos con la mortalidad.

\section{Concordancia del tratamiento antimicrobiano prescrito}

En $239 / 245$ (97,5\%) de los pacientes pudo determinarse la concordancia del tratamiento antimicrobiano prescrito. En el análisis no estratificado, no se observó asociación entre la concordancia y la mortalidad ( $R R=2,23$; IC95\%: 0,39 a 12,7); pero al estratificar por diagnóstico, se observó un riesgo de morir tres veces superior en los pacientes de meningitis que recibieron tratamientos discordantes comparados con los que recibieron tratamientos concordantes $(50 \%$ frente a $17,3 \%)$ $(\mathrm{RR}=2,88$; IC95\%: 1,16 a 7,16). Sin embargo, en los enfermos de neumonía no se presentaron fallecimientos aunque hubieran recibido tratamientos discordantes.

\section{Mortalidad y serotipos de Streptococcus pneumoniae}

El grupo de referencia para el análisis quedó constituido por los tipos capsulares 1, 10A, 12F, 13, 14, 15A, 16F, 18A, 19A, 19B, 19C, 19F, 21, 22F, $24 \mathrm{~F}, 28 \mathrm{~A}, 34,38,4,5,9 \mathrm{~N}$ y $9 \mathrm{~V}(n=146)$. En el cuadro 3 se observan los serotipos que se asociaron con la mortalidad en el análisis bivariado.

No se observaron diferencias estadísticamente significativas con respecto a la distribución de los serotipos por diagnóstico, con excepción del serotipo $23 \mathrm{~F}$ y el serogrupo 6 que se aislaron más frecuentemente en pacientes con meningitis que en los que padecían neumonía $(R R=1,55$; IC95\%: 1,12 a 2,14 y RR = 1,65; IC95\%: 1,25 a 2,19, respectivamente). En los casos de neumonía se observó una notable acentuación de la asociación con la mortalidad de los serotipos $23 \mathrm{~F}(\mathrm{RR}=21,25$; IC95\%: 2,16 a 209,52); $6 \mathrm{~A} / 6 \mathrm{~B}(\mathrm{RR})=7,73$; IC95\%: 0,52 a $114,95)$; y $7 \mathrm{~F}(\mathrm{RR}=42,50$; IC95\%: 3,89 a 464,32$)$.

$\mathrm{Al}$ controlar por resistencia a la penicilina y multirresistencia, se observó que parte del efecto del serotipo 23F sobre la mortalidad se debió a la resistencia a la penicilina (riesgo corregido = 2,95; IC95\%: 0,62 a 12,68) y a la multirresistencia (riesgo corregido $=$ 1,27; IC95\%: 0,18 a 8,75). La asociación del serogrupo 6 con la mortalidad no se modificó por la presencia de resistencia a la penicilina (riesgo corregido $=3,88$; IC95\%: 1,43 a 10,49), ni de multirresistencia (riesgo corregido $=3,42$; IC95\%: 0,94 a 12,51). Entre los demás serotipos asociados con la mortalidad (7F, 8,15B, 18C y 4), no hubo aislamientos con resistencia a la penicilina ni multirresistencia. 
CUADRO 2. Asociación de la resistencia a antimicrobianos de Streptococcus pneumoniae con la mortalidad en casos de neumonía y meningitis en menores de 5 años, Colombia, 1994-1996

\begin{tabular}{|c|c|c|}
\hline Antibiótico & $\begin{array}{c}\text { Meningitis } \\
\text { RR }^{\mathrm{a}} \text { (IC95\%) }\end{array}$ & $\begin{array}{l}\text { Neumonía } \\
\text { RR (IC95\%) }\end{array}$ \\
\hline \multicolumn{3}{|l|}{ Penicilina } \\
\hline $\mathrm{S}^{\mathrm{b}}$ & 1,0 & 1,0 \\
\hline $\mathrm{I}^{\mathrm{c}}$ & $1,35(0,45$ a 4,04$)$ & $4,58(1,10$ a 19,19$)$ \\
\hline$R^{d}$ & $3,91(1,6 \text { a } 9,9)^{e}$ & 0 \\
\hline \multicolumn{3}{|l|}{ Ceftriaxona } \\
\hline$S$ & 1,0 & \\
\hline I & $5,67(3,73$ a 8,62$)$ & 0 \\
\hline $\mathrm{I}+\mathrm{R}$ & $5,67(3,73$ a 8,62$)$ & 0 \\
\hline \multicolumn{3}{|l|}{ Eritromicina } \\
\hline$S$ & 1,0 & \\
\hline I & $3,25(1,55$ a 6,86$)$ & 0 \\
\hline$I+R$ & $3,0(1,40$ a 6,41$)$ & $1,04(0,14$ a 7,86$)$ \\
\hline \multicolumn{3}{|l|}{ TMS } \\
\hline$S$ & 1,0 & 1,0 \\
\hline I & $1,45(0,51$ a 4,12$)$ & $3,88(0,95$ a 15,86$)$ \\
\hline$I+R$ & $1,97(0,91$ a 4,29$)$ & $3,0(0,8$ a 11,29$)$ \\
\hline \multicolumn{3}{|c|}{$\begin{array}{l}\text { Resistencia } \\
\text { (antimicrobianos) }\end{array}$} \\
\hline 0 & 1,0 & 1,0 \\
\hline $1-2$ & $1,45(0,6$ a 3,7$)$ & $2,86(0,60$ a 8,51$)$ \\
\hline $3-5$ & $3,30(1,2 \text { a } 8,2)^{\dagger}$ & 0 \\
\hline
\end{tabular}

a $\mathrm{RR}=$ riesgo relativo

b $\mathrm{S}=$ sensible.

$C \mathrm{I}=$ resistencia intermedia

${ }^{\mathrm{d}} \mathrm{R}=$ alta resistencia.

${ }^{\mathrm{e}} \chi^{2}$ tendencia lineal 3,$31 ; P=0,03$

${ }^{\dagger} \chi^{2}$ tendencia lineal 4,$9 ; P=0,06$.

CUADRO 3. Asociación de los tipos capsulares de Streptococcus pneumoniae con la mortalidad de niños menores de 5 años, Colombia, 1994-1996

\begin{tabular}{lccc}
\hline Serotipo & Fallecidos & Supervivientes & RR (IC95\%) \\
\hline $6 \mathrm{~A} / 6 \mathrm{~B}^{\mathrm{a}}$ & 7 & 31 & $3,84(1,43 \mathrm{a} 10,29)$ \\
$7 \mathrm{~F}$ & 2 & 4 & $6,95(1,82 \mathrm{a} 26,6)$ \\
8 & 1 & 1 & $10,4(2,18 \mathrm{a} 49,7)$ \\
$23 \mathrm{~F}^{\mathrm{a}}$ & 7 & 19 & $5,62(2,15 \mathrm{a} 14,68)$ \\
$35 \mathrm{~B}$ & 1 & 0 & $20,86(10,12 \mathrm{a} 42,97)$
\end{tabular}

a Serotipos que se asociaron con resistencia a la penicilina y multirresistencia. El grupo de referencia estuvo constituido por los serotipos asociados con una mortalidad menor de $10 \%$, que afectaron a 146 pacientes, de los cuales 7 fallecieron y 139 sobrevivieron.

\section{Resultados del análisis multivariado de los factores asociados con la mortalidad}

En el cuadro 4 se presentan las variables que quedaron incluidas en el modelo de regresión logística. El diagnóstico de meningitis y el serotipo 7F fueron los mayores predictores de mortalidad. Se crearon también modelos de regresión logística independientes tanto para los casos de meningitis como para los de neumonía. En los de meningitis, la multirresistencia fue la única variable que permaneció asociada con la mortalidad de forma estadísticamente significativa $(O R=4,33$; $P=0,02)$. En los de neumonía, las va- riables que permanecieron asociadas con la mortalidad de forma estadísticamente significativa fueron la resistencia a TMS $(O R=11,99 ; P=0,02)$ y los serotipos 7F $(O R=80,99 ; P=0,01)$; 15B $(O R=40,49 ; P=0,01)$ y $4(O R=$ $16,19 ; P=0,06)$.

\section{DISCUSIÓN}

A pesar de los progresos logrados en el tratamiento con antimicrobianos y la modernización de los servicios de cuidados intensivos, S. pneumoniae sigue siendo una causa significante de mortalidad y, por lo tanto, constituye un problema importante de salud pública $(4,23,24)$. La letalidad de $11 \%$ observada en este estudio concuerda con los hallazgos de estudios previos en poblaciones infantiles, en los que la mortalidad por enfermedad invasora causada por S. pneumoniae ha variado de 2 a 15\% (9, 25-27). Sin embargo, no se encontraron diferencias estadísticamente significativas en la mortalidad por sexo o grupo de edad, aunque en la literatura se ha informado de un incremento significativo de las muertes en varones y menores de 2 años en el período de 1969 a $1978(19,25)$.

La letalidad por meningitis fue de $19 \%$, similar a la hallada en estudios previos $(19,26,28)$. Asimismo, el diagnóstico de meningitis fue el que más se

CUADRO 4. Resultados del análisis multivariado de los factores asociados con la mortalidad por Streptococcus pneumoniae en niños menores de 5 años, Colombia, 1994-1996

\begin{tabular}{lrc}
\hline \multicolumn{1}{c}{ Variable } & OR & Valor $P$ \\
\hline $\begin{array}{l}\text { Examen físico de ingreso } \\
\quad \text { Polipnea }\end{array}$ & 2,74 & 0,03 \\
$\begin{array}{l}\text { Diagnóstico } \\
\quad \text { Meningitis }\end{array}$ & 5,02 & 0,0001 \\
$\begin{array}{l}\text { Resistencia a } \\
\text { antimicrobianos }\end{array}$ & & \\
$\quad$ TMS \\
$\begin{array}{l}\text { Serotipos: } \\
\quad \text { 7F }\end{array}$ \\
$\quad 2$
\end{tabular}


asoció con la mortalidad, lo cual también coincide con los estudios que muestran un riesgo incrementado de muerte en pacientes con meningitis por S. pneumoniae, comparada con otros casos de enfermedad invasora por la misma bacteria o con meningitis de otras causas $(19,24-27,29)$.

Se ha observado que las condiciones críticas en que se encuentra el paciente en el momento de ingreso a los centros de salud así como los procesos patológicos subyacentes determinan en gran medida la evolución de los pacientes con enfermedad invasora por $S$. pneumoniae $(24,30,31)$. En este estudio, las variables anotadas durante el examen físico de ingreso que más se asociaron con la mortalidad fueron la polipnea y la hipotermia, aunque esta última no alcanzó significación estadística. Los estados patológicos subyacentes no tuvieron ninguna asociación significativa con la mortalidad.

El impacto de la resistencia a antimicrobianos en la evolución y la mortalidad de pacientes con enfermedad invasora por $S$. pneumoniae no ha sido claramente establecido (8-10, 31-33), pero se ha definido mejor en la meningitis (34) que en la neumonía $(35,36)$, tal como ocurrió en el presente estudio. En relación con la meningitis, el análisis bivariado indicó una asociación de la mortalidad con la resistencia a penicilina, ceftriaxona, eritromicina y con los tratamientos antimicrobianos discordantes. Sin embargo, en el análisis multivariado estas asociaciones desaparecieron y solo la multirresistencia permaneció asociada significativamente con la mortalidad.

En cuanto a la neumonía, la resistencia a TMS tuvo el mayor impacto sobre la mortalidad. Este hallazgo es importante, ya que dicho antibiótico es uno de los agentes de elección para el tratamiento de la neumonía en niños a nivel comunitario (37). Cabe destacar que su efecto sobre la mortalidad fue independiente de la asociación con resistencia a la penicilina o multirresis- tencia y de las demás variables analizadas en el presente estudio.

En lo referente a los tipos capsulares de S. pneumoniae, entre ellos se han documentado diferencias de virulencia, habilidad para adquirir un fenotipo de resistencia a antimicrobianos $(16,38$, 39) y evolución de los pacientes (12, 13). En el presente estudio se observó que los serotipos 7F y 8 tuvieron el mayor impacto sobre la mortalidad por S. pneumoniae de tipo invasor, pero no se asociaron con resistencia a la penicilina ni con multirresistencia y su efecto fue independiente de las demás variables evaluadas. Esos serotipos han sido identificados como más virulentos e inmunógenos, y con menor potencial de desarrollo de resistencia a los antimicrobianos $(38,40)$, lo cual hace pensar que su asociación con la mortalidad se relaciona directamente con su virulencia intrínseca.

En los casos de neumonía, específicamente, se observó que los serotipos fueron los predictores de mortalidad más importantes junto con la resistencia al TMS. El hecho de que el impacto de los serotipos se haya visto reflejado en la neumonía sugiere que la virulencia de estos se observa más en los cuadros clínicos menos graves que en aquellos que conllevan un riesgo implícito incrementado de mortalidad, como sucede en la meningitis.

Cabe destacar que, de los tipos capsulares que se asociaron con mortalidad en el análisis bivariado, solamente el 6 y el 23F presentaron aislamientos con resistencia a la penicilina y multirresistencia. De hecho, el efecto del serotipo $23 \mathrm{~F}$ sobre la mortalidad se debió sobre todo a la multirresistencia y en parte a la resistencia a la penicilina, y no al serotipo como tal. Sin embargo, el efecto del serogrupo 6 en la mortalidad fue independiente de esas condiciones, si bien su asociación con la mortalidad desapareció en el análisis multivariado.

Actualmente, la Iniciativa en pro de la Vacuna Infantil está trabajando a nivel mundial con el fin de elaborar una vacuna que conjugue polisacáridos de $S$. pneumoniae con proteínas para incrementar su inmunogenicidad en los menores de 2 años. Se espera que la vacuna incluya de 9 a 11 de los serotipos más comunes del mundo. De acuerdo con la estimación de la Iniciativa, se podrían prevenir 580000 muertes al año por neumonía y meningitis ocasionadas por $S$. pneumoniae al producirse inmunidad heredada. Aun si este fenómeno no se manifestara, se prevendrían al menos 400000 muertes anuales (14).

Los resultados de este estudio sugieren que el impacto de una vacuna contra infecciones por $S$. pneumoniae puede maximizarse si al elaborarla se tienen en cuenta los criterios de virulencia de los serotipos más la frecuencia con que desarrollan resistencia a los antimicrobianos. Sin embargo, este estudio presenta limitaciones en la forma como fueron medidos los estados patológicos subyacentes y los hallazgos clínicos al ingreso, ya que esa información faltaba en un número de las historias clínicas consultadas. Sin embargo, no hubo diferencias estadísticamente significativas entre los grupos con datos y sin ellos en cuanto a las variables epidemiológicas básicas como edad, sexo y tasa de mortalidad, por lo que no puede inferirse que sean poblaciones diferentes y que las asociaciones con la mortalidad difieran entre ellas. De todas maneras, deberían realizarse estudios prospectivos para investigar específicamente esas asociaciones, con el fin de validar los hallazgos aquí presentados y ampliar el criterio de selección de los serotipos para las vacunas.

Agradecimiento. Los autores expresan su agradecimiento a José Luis Di Fabio, coordinador del proyecto Sistema Regional de Vacunas (SIREVA) de la OPS, por su apoyo incondicional. 


\section{REFERENCIAS}

1. Gray BM, Dillon Jr HC. Clinical and epidemiologic studies of pneumococcal infections in children. Pediatr Infect Dis J 1986;5(2):201-207.

2. Klein JO. The epidemiology of pneumococcal disease in infants and children. Rev Infect Dis 1981;3(2):246-253.

3. Finland M. Pneumococcal infections. En: Evans AS, Feldman HA, eds. Bacterial infections of humans: epidemiology and control. New York: Plenum Medical Books; 1982. pp. 417-433.

4. Garenne M, Ronsmaus C, Campbell H. The magnitude of mortality from acute respiratory infections in children under 5 years in developing countries. World Health Stat Q 1992;45:180-191.

5. Appelbaum PC. Antimicrobial resistance in Streptococcus pneumoniae: an overview. Clin Infect Dis 1992;15(1):77-83.

6. Klugman KP. Pneumococcal resistance to antibiotics. Clin Microbiol Rev 1990;3(2): 171-196.

7. Pallares R, Gudiol F, Linares J, Ariza J, Rufi G, Murgui L, et al. Risk factors and response to antibiotic therapy in adults with bacteremic pneumonia caused by penicillin-resistant pneumococci. N Engl J Med 1987;317(1):18-22.

8. Koornhof HJ, Wasas A, Klugman K. Antimicrobial resistance in Streptococcus pneumoniae: a South African perspective. Clin Infect Dis 1992;15(1):84-94.

9. Garcia Leoni ME, Cercenado E, Rodeño P, Bernaldo de Quirós JCL, MartínezHernández D, Bouza E. Susceptibility of Streptococcus pneumoniae to penicillin: a prospective microbiological and clinical study. Clin Infect Dis 1992;14(2):427-435.

10. Istre GR, Tarpay M, Anderson M, Pryor A, Welch D. Invasive disease due to Streptococcus pneumoniae in an area with a high rate of relative penicillin resistance. J Infect Dis 1987;156(5):732-735.

11. Austrian R. Pneumococcal infections. En: Last JM, Wallace RB. Public health and preventive medicine. Norwalk Connecticut: Appleton Century Crofts; 1992: 87-89.

12. Hsueh PR, Wu JJ, Hslue TR. Invasive Streptococcus pneumoniae infection associated with rapidly fatal outcome in Taiwan. J Formos Med Assoc 1996;95(5): 364-371.

13. Calder MA, McHardy VU, Schonell ME. Importance of pneumococcal typing in pneumonia. Lancet 1970;1(7636):5-7.

14. Gray BM, Converse III GM, Dillon HC Jr. Serotypes of Streptococcus pneumoniae causing disease. J Infect Dis 1979;140(6): 979-983.

15. New vaccines: a pneumococcal vaccine to save children of all ages nears final testing. Children's Vaccine Initiative Forum 1996;13:3-11.

16. Montgomery JM, Lehmann D, Smith $T$, Michael A, Joseph B, Lupiwa T, et al. Bac- terial colonization of upper tract and its association with acute lower tract infections in high-land children of Papua, New Guinea. Rev Infect Dis 1990;12 (Suppl 8):S1006-1016.

17. Castañeda E, Leal AL, Castillo O, De la Hoz F, Vela MC, Arango M, et al. Distribution of capsular types and antimicrobial susceptibility of invasive isolates of Streptococcus pneumoniae in Colombian Children. Microb Drug Resist 1997; 3(2): 147-152.

18. World Health Organization, Program for the Control of Acute Respiratory Infections. Technical bases for WHO recommendations on the management of pneumonia in children at first level health facilities. Geneva; 1991. (WHO/ARI/9120).

19. Karup Pedersen F, Henrichsen J. Pneumococcal meningitis and bacteremia in Danish children 1969-1978. Acta Path Immunol Scand 1983;Sect B 91:129-134.

20. Londoño JL. Análisis estratificado: metodologia de la investigación epidemiológica. Editorial Universidad de Antioquia; 1995.

21. Dean AG, Dean JA, Burton AH, et al. Epi Info Version 6.0: a word processing, database and statistics program for epidemiology on microcomputers. Atlanta, Georgia: Centers for Disease Control and Prevention; 1994

22. Norusis MJ. SPSS Base para Windows, version 6.0. SPSS Inc; 1996.

23. Raffin TA. Intensive care unit survival of patients with systemic illness. Am Rev Respir Dis 1989;140(2, part 2):S28-S35.

24. Burman LA, Norrby R, Trollfors B. Invasive pneumococcal infections: incidence, predisposing factors, and prognosis. Rev Infect Dis 1985;7(2):133-142.

25. Dagan R, Englehard D, Piccard E. Epidemiology of invasive childhood pneumococcal infections in Israel. The Israeli Pediatric Bacteremia and Meningitis Group. JAMA 1992;268(23):3328-3332.

26. Davidson M, Parkinson AJ, Bulkow LR Fitzgerald MA, Peters HV, Parks D. The epidemiology of invasive pneumococcal disease in Alaska, 1986-1990: ethnic differences and opportunities for prevention. J Infect Dis 1994;170(2):368-376.

27. Jacobs NM, Lerdkachornsuk S, Metzger WI. Invasive pneumococcal bacteremia in infants and children: a ten-year experience at the Cook County Hospital with special reference to the pneumococcal serotypes isolated. Pediatrics 1979;64(3): 296-300.

28. Kornelisse RF, Westerbeek CML, Spoor $A B$, van de Heijde B, Spanjaard L, Neijens HJ, de Groot R. Pneumoccal meningitis in children: prognostic indicators and outcome. Clin Infect Dis 1995;21(6): 1390-1397.

29. Ford $\mathrm{H}$, Wright J. Bacterial meningitis in Swaziland: an 18 month prospective study and its impact. J Epidemiol and Community Health 1994;48(3):276-280.

30. Bennet NM, Buffington J, Marc La Force F. Pneumococcal bacteremia in Monroe County, New York. Am J Public Health 1992;82(11):1513-1516.

31. Pallares R, Liñares J, Vadillo M, Cabellos C, Manresa F, Viladrich PF, et al. Resistance to penicillin and cephalosporin and mortality from severe pneumococcal pneumonia in Barcelona, Spain. N Engl J Med 1995;333(8):474-480.

32. Tan TQ, Mason EO, Kaplan SL. Systemic infections due to Streptococcus pneumoniae relatively resistant to penicillin in a children's hospital: clinical management and outcome. Pediatrics 1992;90(6):928-933.

33. Frankel RE, Virata M, Hardalo C, Altice FL, Friedland G. Invasive pneumococcal disease: clinical features, serotypes, and antimicrobial resistance patterns in cases involving patients with and without human inmunodeficiency virus infections. Clin Infect Dis 1996;23(3):577-584.

34. Sloas MM, Barrett FF, Chesney PJ et al. Cephalosporin treatment failure in penicillin-and cephalosporin-resistant Streptococcus pneumoniae meningitis. Pediatr Infect Dis J 1992;11(8):662-666.

35. Finch RG. Pneumonia: the impact of antibiotic resistance on its management. Microb Drug Resist 1995;1(2):149-158.

36. Friedland IR. Comparison of the response to antimicrobial therapy of penicillin resistant and pencillin susceptible pneumococcal disease. Pediatr Infect Dis J 1995; 14(10):885-890.

37. Colombia, Ministerio de Salud, y Organización Panamericana de la Salud. Atención del niño con infección respiratoria aguda. MS, OPS; 1992. (Serie Paltex no. 21).

38. Bédos JP, Chevret S, Chastang C, Geslin P, Régnier B. Epidemiological features of and risk factors for infection by Streptococcus pneumoniae strains with diminished susceptibility to penicillin: findings of a French survey. Clin Infect Dis 1996;22(1): 63-72.

39. Briles DE, Crain MJ, Gray BM, Forman C, Yother J. Strong association between capsular types and virulence for mice among human isolates of Streptococcus pneumoniae. Infect Immun 1992;60(1):111-116.

40. Fenoll A, Bourgon CM, Muñoz R, Vicioso D, Casal J. Serotype distribution and antimicrobial resistance of Streptococcus pneumoniae isolates causing systemic infections in Spain, 1979-1989. Rev Infect Dis 1991;13(1):56-60.

Manuscrito recibido el 21 de enero de 1998 y aceptado para publicación, tras revisión, el 21 de diciembre de 1998 
ANEXO 1. Grupo Colombiano de Trabajo en Streptococcus pneumoniae

Centros en Santa Fe de Bogotá:

Grupo de Microbiología, Instituto Nacional de Salud: MC Vela; Hospital Universitario de La Misericordia: M Arango, J Serrato, MR Navarrete, M García, C Lozada; Hospital Infantil Lorencita Villegas de Santos: ME Gama; Hospital de Santa Clara: M Calle, G Aristizábal, A Tovar; Clínica del Niño, ISS: C Paredes, A Arenas.

Clínica de Colsubsidio, Hospital de Kennedy, Hospital Simón Bolívar, Hospital San Blas, Clínica CAFAM, Hospital del Guavio, Hospital de Bosa, Hospital La Granja, Hospital del Tunal, Hospital La Victoria, Laboratorio Central Secretaría de Salud, Fundación Clínica Shaio e Instituto Roosevelt.

\section{Centros en Medellín:}

Corporación para Investigaciones Biológicas: H Trujillo, GI Mejía, N Correa, J. Robledo; Hospital Infantil: ML Valencia, W Parra, N Agudelo, C Zapata; Hospital Pablo Tobón Uribe: S Jaramillo, C. Suárez; Hospital General: F. Montoya, V. García.

\section{Centros en Cali:}

Hospital Universitario del Valle: A Levy, H Porras, A Sánchez, LM Gallardo, N Villamarín, AL Bohorquez, P López; Hospital Infantil Club Noel: D Saa, A Moreno, J. Guerrero.

Centro de referencia internacional:

National Center for Streptococcus (NCS) Alberta, Canadá: M Lovgren, J Weekes, J Talbot.

ABSTRACT Severe pneumonia and meningitis caused by Streptococcus pneumoniae have been persistently associated with high mortality rates, despite advances in antimicrobial therapy and the development of vaccines. Resistance to penicillin and other antimicrobial agents is increasing and spreading worldwide. Even though risk factors for development of antimicrobial resistance have been identified, their influence on mortality has not been clarified. With regard to virulence, differences among serotypes have been determined, but their impact on mortality is unknown. The aim of this study was to determine the risk factors associated with mortality in children with invasive pneumococcal disease. Clinical records for 245 children under 5 years of age with invasive disease due to $S$. pneumoniae were reviewed. Children were diagnosed between 1994 and 1996 in Colombia, during the study of S. pneumoniae capsular types conducted by the Pan American Health Organization's Regional System for Vaccines. Of the 245 patients whose charts were examined, 29 (11\%) died. No significant differences in age, gender, underlying disease, nor antimicrobial treatment concordance were found. Variables associated with mortality in the univariate analysis were a diagnosis of meningitis; antimicrobial resistance to penicillin, trimethoprim-sulfamethoxazole (TMS), or erythromycin; multiresistance, and serotypes $6,23 \mathrm{~F}, 7 \mathrm{~F}, 8$, and 35B. In the logistic regression, serotypes $7 \mathrm{~F}(\mathrm{OR}=7,13 ; P=0,04)$ and $8(\mathrm{OR}=13,8 ; P=0,07)$, polipnea $(\mathrm{OR}=2,74 ; P=0,03)$, meningitis $(\mathrm{OR}=5,02 ; P=0,0001)$ and TMS resistance $(\mathrm{OR}=2,62 ; P=0,02)$ continued to be associated with mortality. In patients with pneumonia, serotype was the factor most consistently associated with mortality; in meningitis patients, it was antimicrobial resistance. Differences in mortality according to serotype must be taken into account in developing a vaccine if a substantial impact on pneumococcal disease morbidity and mortality is to be achieved. 